\title{
Constructive Interference Beamforming for Cooperative Dual-Hop MIMO Relay Systems
}

\author{
(Invited Paper) \\ Ang Li and Christos Masouros \\ Dept. of Electronic and Electrical Eng., University College London, London, UK \\ Email: \{ang.li.14, c.masouros\}@ucl.ac.uk
}

\begin{abstract}
In this paper, we consider the downlink transmission for a dual-hop amplify-and-forward (AF) multiple-antenna relay systems, where we propose beamforming techniques for interference exploitation on a symbol level. Based on the constructive interference $(\mathrm{CI})$, we firstly propose a joint source/relay precoding, where the precoding matrices at the source and the output signals at the relay are jointly optimized. To alleviate the high computational costs and circumvent the difficulty of practical implementation of the joint design, we further propose a low-complexity decoupled approach, where a closed-form linear precoding method is first employed at the source, and we then optimize the beamforming matrix at the relay for interference exploitation. It is revealed by numerical results that the proposed approaches that exploit the instantaneous interference can achieve an improved performance over the conventional case with a linear approach.

Index Terms - Cooperative systems, dual-hop AF relay, MIMO, constructive interference, optimization.
\end{abstract}

\section{INTRODUCTION}

Due to the significant multiplexing gain and diversity gain, multiple-input multiple-output (MIMO) techniques have been widely employed to support the exponential growth in both the wireless devices and the wireless data traffic [1]-[3]. Among MIMO techniques, relaying and cooperative multi-antenna systems have emerged as key advocates due to the promising potential to improve the system capacity and increase reliable coverage, especially when the direct links between the base station (BS) and the users experience deep fading [4]. Motivated by these benefits, extensive research efforts have been devoted to the cooperative relay systems [5]-[9]. Specifically, the designs of amplify-and-forward (AF) and decode-andforward (DF) relaying have been well studied for the system consisting of one source and one destination through one relay in [5]-[7], where the capacity bounds are theoretically studied in [5] for the non-regenerative MIMO relay systems. The rate and error performance have been studied in [6][7] for the relay-assisted cooperative systems. Multiple relays have been considered in [8][9], where the capacity scaling law is analyzed in [8], while a relay selection method has been introduced in [9] for additional diversity benefits.

In addition, precoding designs have also been widely studied for the relay-aided communication systems [10]-[16]. In [10][11], the authors consider the maximization of the system capacity, where [10] considers both the case with a direct link and the case without a direct link, while in [11] three distinct scenarios are considered with respect to the availability of channel state information (CSI) at the relay. [12] considers the maximum signal-to-interference-plus-noise ratio (MSINR) criterion, where it is shown that the optimality is achieved when the equivalent source-relay-destination channel is diagonalized into sub-channels. Joint source/relay precoding has been considered in [13][14], where in [13] the precoding method has been designed for a MIMO two-way relay systems based on mean-squared-error (MSE) criterion, while [14] studies the case with limited feedback for the dual-hop MIMO relay systems, and focuses on the selection process for the successive interference cancellation (SIC) at the receiver. The study of limited feedback has been extended to the case of multiuser in [15], where a linear precoding scheme is considered at the relay and the upper bound on the rate loss due to the quantization is characterized. The maximization of the energy efficiency in a multiuser MIMO relay system is considered in [16]. Nevertheless, all the above precoding designs may be sub-optimal as they ignore the fact that interference can be exploited on an instantaneous basis [18]-[25]. It is shown that on the symbol level, the instantaneous interference can be classified into constructive and destructive [18], and downlink optimization-based transmission techniques have further been proposed such that all the interference is manipulated to be constructive. Such interference exploitation methods have not been well studied for the relay-aided cooperative communication systems.

Therefore in this paper, we consider the symbol-level precoding techniques for the multiple-antenna cooperative dualhop AF relay systems for interference exploitation. We first focus on a joint source/relay precoding technique, where the optimization problem is constructed based on the geometry of the modulation constellations to exploit the constructive interference. Noting that the formulated optimization is biconvex, we propose an alternating optimization process to iteratively update the precoding matrices at the BS and the relay, where the proposed alternating approach is guaranteed to converge. Nevertheless, this joint approach is computationally expensive and moreover requires additional information exchange between the BS and relay. Accordingly, we further propose a decoupled precoding design, where for simplicity the BS designs its precoding matrix following the closed-form zero-forcing (ZF) or regularized ZF (RZF) [26] approach, and the relay subsequently designs its precoding matrix based on 
the CI formulation. It is shown by numerical results that both of the proposed joint and decoupled approach can achieve an improved performance over the conventional closed-form precoding at the relay, while the decoupled method achieves a similar performance to the complicated joint scheme, which favours its usefulness in practical cooperative systems.

The rest of this paper is organized as follows: Section II gives a brief introduction of the system model, and Section III includes the detailed description of the proposed joint and decoupled precoding methods. The numerical results are shown in Section IV, and Section V concludes our paper.

Notations: $a$, a, and A denote scalar, vector and matrix, respectively. $(\cdot)^{-1}$ and $(\cdot)^{H}$ denote inverse and conjugate transposition of a matrix, respectively. $|\cdot|$ denotes the modulus, and $\|\cdot\|$ denotes the Frobenius norm, respectively. $\mathcal{C}^{n \times n}$ represents an $n \times n$ matrix in the complex set, and $\mathbf{I}$ denotes the identity matrix.

\section{SySTEM MODEL}

We consider a cooperative MIMO broadcast system with an AF relay in the downlink, as shown in Fig. 1. The BS with $N_{t}$ antennas communicates with a total number of $K$ singleantenna users through a dedicated relay, where we assume that there are no available direct links between the BS and the users due to the severe pathloss and shadowing effect. The relay employs a total number of $N_{R}$ antennas, and we assume $N_{R} \leq N_{t}$ to facilitate precoding at the BS [17]. In the following, we focus on the extreme case where $K=N_{R}$, while in the case where $K>N_{R}$ a user scheduling is required prior to transmission. We denote the channel matrix between the BS and the relay as $\mathbf{H}_{s r} \in \mathcal{C}^{N_{R} \times N_{t}}$, and the channel between the relay and the users as $\mathbf{H}_{r d} \in \mathcal{C}^{K \times N_{R}}$, respectively. Without loss of generality, we employ an uncorrelated flat-fading Rayleigh channel in this paper. We express the data symbol vector as $\mathbf{s}=\left[s_{1}, s_{2}, \cdots, s_{K}\right]^{T}$ that is from a normalized PSK modulation, while the interference exploitation introduced in this paper trivially extends to QAM modulations [22]. In Phase I, the symbols are multiplexed by a source precoding matrix $\mathbf{F}_{s} \in \mathcal{C}^{N_{t} \times K}$ and then transmitted to the relay. Accordingly, we express the received signal vector at the relay as

$$
\mathbf{y}_{r}=\mathbf{H}_{s r} \mathbf{F}_{s} \mathbf{s}+\mathbf{n}_{r}
$$

where $\mathbf{y}_{r} \in \mathcal{C}^{N_{R F} \times 1}$ denotes the received signal vector, and $\mathbf{n}_{r} \sim \mathcal{C} \mathcal{N}\left(\mathbf{0}, \sigma_{r}^{2} \cdot \mathbf{I}\right)$ represents the noise vector at the relay. As a symbol-level constructive interference beamforming is considered in this paper, for coherence a symbol-level power constraint in enforced on $\mathbf{F}_{s}$ that satisfies

$$
\left\|\mathbf{F}_{s} \mathbf{s}\right\| \leq P_{s}
$$

where $P_{s}$ denotes the maximum transmit power available at the BS. In Phase II, the relay employs a transmit beamforming

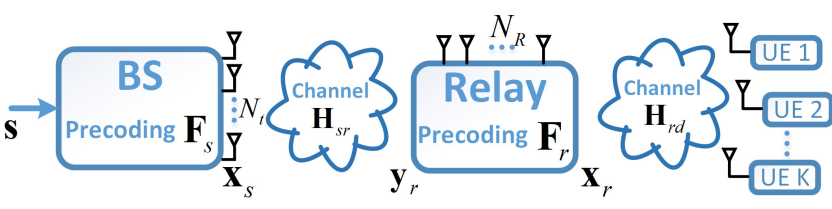

Fig. 1: Block diagram of dual-hop MIMO relay systems

technique and forward the received signal at Phase I to the users, expressed as

$$
\begin{aligned}
\mathbf{r}_{d} & =\mathbf{H}_{r d} \mathbf{x}_{r}+\mathbf{n}_{d} \\
& =\mathbf{H}_{r d} \mathbf{F}_{r} \mathbf{y}_{r}+\mathbf{n}_{d} \\
& =\mathbf{H}_{r d} \mathbf{F}_{r}\left(\mathbf{H}_{s r} \mathbf{F}_{s} \mathbf{s}+\mathbf{n}_{r}\right)+\mathbf{n}_{d},
\end{aligned}
$$

where $\mathbf{r}_{d}$ denotes the received signals for the users, and $\mathbf{n}_{d} \sim$ $\mathcal{C N}\left(\mathbf{0}, \sigma_{d}^{2} \cdot \mathbf{I}\right)$ represents the corresponding noise vector at the user side. In Phase II, the power constraint on the relay is expressed as

$$
\begin{aligned}
& \left\|\mathbf{F}_{r} \mathbf{y}_{r}\right\| \leq P_{r}, \\
\Rightarrow & \left\|\mathbf{F}_{r}\left(\mathbf{H}_{s r} \mathbf{F}_{s} \mathbf{s}+\mathbf{n}_{r}\right)\right\| \leq P_{r}
\end{aligned}
$$

where $P_{r}$ denotes the maximum transmit power for the relay.

\section{Proposed Transmit BeAmForming FOR INTERFERENCE EXPLOITATION}

In this section, we propose the joint source/relay and lowcomplexity decoupled precoding methods for the considered cooperative systems based on the formulation of CI. CI is defined as the interference that pushes the received signals away from the detection thresholds [18]-[22]. The exploitation of CI was firstly introduced in [18] to improve the performance of the $\mathrm{ZF}$ precoding, and was more recently extended to the optimization-based approaches for PSK modulations in [19] as well as QAM modulations in [22]. It is demonstrated that, as long as the resulting interfered signal is located in the constructive region of the modulation constellation, this increases the distance between the received signal and the detection thresholds, and an improved performance can be obtained. In what follows, we present the joint and decoupled source/relay precoding designs based on CI.

\section{A. Joint Source/Relay Design}

Before we construct the optimization problem for the joint source/relay design, we first obtain the CI formulation based on the geometry of the modulation constellations, where we employ one quarter of a normalized QPSK modulation as the example, depicted in Fig. 2. As can be observed, as long as the node B that represents the noiseless received signal lies in the constructive region, its Euclidean distance to both detection thresholds is increased. Specific to our problem in this paper, we denote

$$
\overrightarrow{O A}=t s_{k}, \quad \overrightarrow{O B}=\mathbf{h}_{r d}^{k} \mathbf{F}_{r} \mathbf{y}_{r}=\lambda_{k} s_{k}
$$

where we have decomposed $\mathbf{H}_{r d}$ into

$$
\mathbf{H}_{r d}=\left[\left(\mathbf{h}_{r d}^{1}\right)^{T},\left(\mathbf{h}_{r d}^{2}\right)^{T}, \cdots,\left(\mathbf{h}_{r d}^{K}\right)^{T}\right]^{T} .
$$




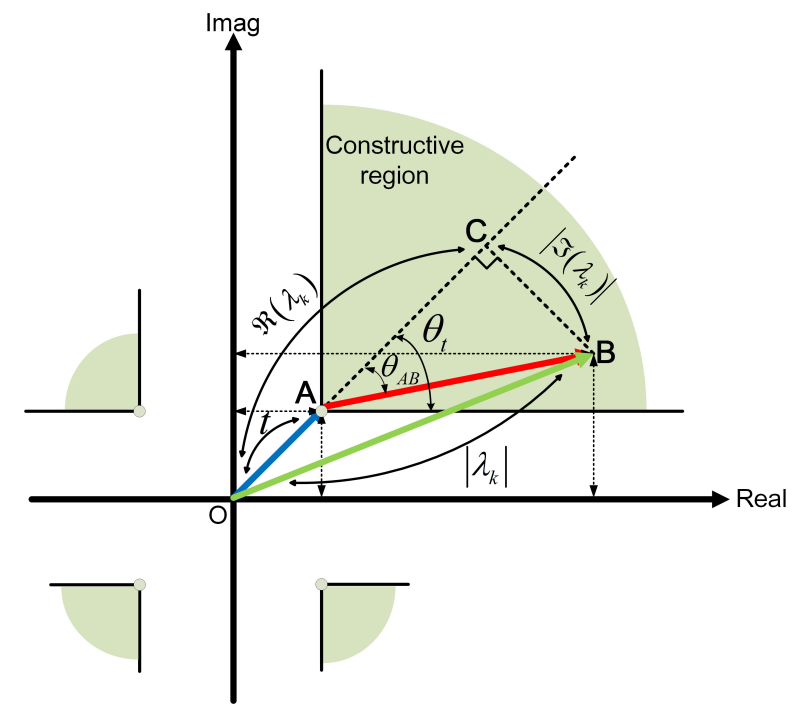

Fig. 2: CI and constructive region for QPSK

In (5), $t$ is a real variable that represents how far the constructive region is pushed away from the detection thresholds, as shown in Fig. 2, and $s_{k}$ is the data symbol for the $k$-th user. OB denotes the noiseless received signal at user $k$, and $\lambda_{k}$ is an introduced complex variable that represents the effect of interference. Based on Fig. 2 and the CI formulation in [19], we obtain that to have node ' $\mathrm{B}$ ' in the constructive region is equivalent to

$$
\begin{aligned}
& \tan \theta_{A B} \leq \tan \theta_{t} \\
\Rightarrow & \frac{|C B|}{|A C|} \leq \tan \theta_{t} \\
\Rightarrow & \frac{\left|\Im\left(\lambda_{k}\right)\right|}{\Re\left(\lambda_{k}\right)-t} \leq \tan \theta_{t} \\
\Rightarrow & {\left[\Re\left(\lambda_{k}\right)-t\right] \tan \theta_{t} \geq\left|\Im\left(\lambda_{k}\right)\right|, }
\end{aligned}
$$

where we note that $\left|s_{k}\right|=1, \forall k \in \mathcal{K}, \mathcal{K}=\{1,2, \cdots, K\}$. The angle threshold can be obtained as $\theta_{t}=\pi / \mathcal{M}$ for $\mathcal{M}$-PSK modulations based on the geometry.

Accordingly, we construct the joint source/relay optimization problem that maximizes $t$ such that the constructive region is pushed as far away as possible, given by

$$
\begin{array}{ll}
\mathcal{P}_{1}: & \max _{\mathbf{F}_{s}, \mathbf{F}_{r}} t \\
\text { s.t. } & \mathbf{h}_{r d}^{k} \mathbf{F}_{r}\left(\mathbf{H}_{s r} \mathbf{F}_{s} \mathbf{s}+\mathbf{n}_{r}\right)=\lambda_{k} s_{k}, \forall k \in \mathcal{K} \\
& {\left[\Re\left(\lambda_{k}\right)-t\right] \tan \theta_{t} \geq\left|\Im\left(\lambda_{k}\right)\right|, \forall k \in \mathcal{K}} \\
& \left\|\mathbf{F}_{s} \mathbf{s}\right\| \leq P_{s} \\
& \left\|\mathbf{F}_{r}\left(\mathbf{H}_{s r} \mathbf{F}_{s} \mathbf{s}+\mathbf{n}_{r}\right)\right\| \leq P_{r}
\end{array}
$$

$\mathcal{P}_{1}$ in its current form is not convex due to the multiplication of the variables. Nevertheless, we note that $\mathcal{P}_{1}$ is bi-convex, i.e. it is convex with respect to $\mathbf{F}_{s}$ when $\mathbf{F}_{r}$ is fixed and vice verse, which motivates the use of an alternating optimization approach, described in the following.
When $\mathbf{F}_{s}$ is fixed, based on $\mathcal{P}_{1}$ the optimization problem to obtain $\mathbf{F}_{r}$ can be transformed into

$$
\begin{array}{ll}
\mathcal{P}_{2}: & \max _{\mathbf{F}_{r}} t \\
\text { s.t. } & \mathbf{h}_{r d}^{k} \mathbf{F}_{r}\left(\mathbf{H}_{s r} \mathbf{F}_{s} \mathbf{s}+\mathbf{n}_{r}\right)=\lambda_{k} s_{k}, \forall k \in \mathcal{K} \\
& {\left[\Re\left(\lambda_{k}\right)-t\right] \tan \theta_{t} \geq\left|\Im\left(\lambda_{k}\right)\right|, \forall k \in \mathcal{K}} \\
& \left\|\mathbf{F}_{r}\left(\mathbf{H}_{s r} \mathbf{F}_{s} \mathbf{s}+\mathbf{n}_{r}\right)\right\| \leq P_{r}
\end{array}
$$

which is a convex optimization and can be efficiently solved via optimization tools such as CVX [27].

When $\mathbf{F}_{r}$ is fixed, the optimization on $\mathbf{F}_{s}$ can be transformed based on $\mathcal{P}_{1}$ into

$$
\begin{array}{ll}
\mathcal{P}_{3}: & \max _{\mathbf{F}_{s}} t \\
\text { s.t. } & \mathbf{h}_{r d}^{k} \mathbf{F}_{r}\left(\mathbf{H}_{s r} \mathbf{F}_{s} \mathbf{s}+\mathbf{n}_{r}\right)=\lambda_{k} s_{k}, \forall k \in \mathcal{K} \\
& {\left[\Re\left(\lambda_{k}\right)-t\right] \tan \theta_{t} \geq\left|\Im\left(\lambda_{k}\right)\right|, \forall k \in \mathcal{K}} \\
& \left\|\mathbf{F}_{s} \mathbf{s}\right\| \leq P_{s} \\
& \left\|\mathbf{F}_{r}\left(\mathbf{H}_{s r} \mathbf{F}_{s} \mathbf{s}+\mathbf{n}_{r}\right)\right\| \leq P_{r}
\end{array}
$$

which is also convex and can be efficiently solved as there is only one variable $\mathbf{F}_{s}$. Based on the above, an alternating optimization algorithm can be constructed to alternately update $\mathbf{F}_{s}$ and $\mathbf{F}_{r}$, given in Algorithm 1, where $N_{\max }$ denotes the maximum iteration number, $\delta$ denotes the convergence accuracy, and $\delta_{t h}$ is the accuracy threshold.

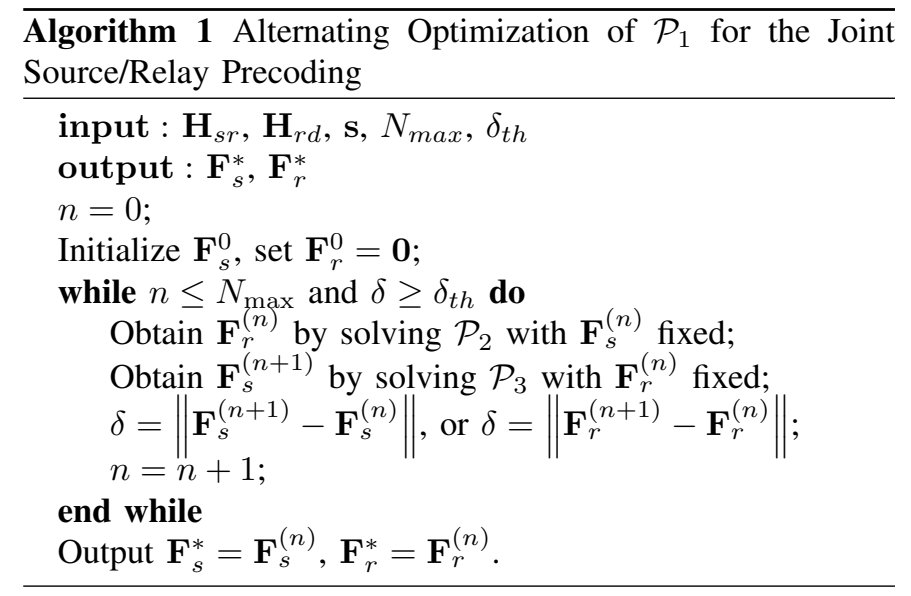

Convergence: In Algorithm 1, we observe that both subproblems $\mathcal{P}_{2}$ and $\mathcal{P}_{3}$ share the same objective function that is to maximize $t$, and both sub-problems are convex and optimally solved. Therefore, the proposed alternating optimization is guaranteed to converge [28], which will also be validated via numerical results where it is shown that Algorithm 1 converges within several iterations.

Remark: While the proposed joint source/relay beamforming can be optimally solved, this technique has the following drawbacks. Firstly, it requires information exchange between the BS and the relay as both channel knowledge $\mathbf{H}_{s r}$ and $\mathbf{H}_{r d}$ is required. Moreover, due to the nature of alternating optimization, the resulting problem is computationally inefficient. Both of the above make it difficult for the joint beamforming to be implemented in practical systems, which motivates us 
to propose the following decoupled approach. Moreover, it is shown in Section IV that the low-complexity decoupled precoding design achieves a similar performance to the joint source/relay precoding method.

\section{B. Low-Complexity Decoupled Design}

We proceed to introduce the low-complexity decoupled design, where the BS designs its precoding matrix solely dependent on the channel between BS and the relay, while the relay designs its precoding matrix based on the received signals and the channel between the relay and users. To be more specific, in Phase I for simplicity the BS employs a closed-form ZF or RZF precoding approach [26], given by

$$
\begin{aligned}
\mathbf{F}_{s}^{Z F} & =\frac{1}{f} \cdot \mathbf{H}_{s r}^{H}\left(\mathbf{H}_{s r} \mathbf{H}_{s r}^{H}\right)^{-1} \\
\mathbf{F}_{s}^{R Z F} & =\frac{1}{f} \cdot \mathbf{H}_{s r}^{H}\left(\mathbf{H}_{s r} \mathbf{H}_{s r}^{H}+K \sigma_{r}^{2} \cdot \mathbf{I}\right)^{-1},
\end{aligned}
$$

where $f$ is the power scaling factor that satisfies (2). Subsequently, in Phase II with the obtained signal vector $\mathbf{y}_{r}$ as shown in (1), the optimization on the precoding matrix $\mathbf{F}_{r}$ at the relay can be expressed as

$$
\begin{array}{ll}
\mathcal{P}_{4}: & \max _{\mathbf{F}_{r}} t \\
\text { s.t. } & \mathbf{h}_{r d}^{k} \mathbf{F}_{r} \mathbf{y}_{r}=\lambda_{k} s_{k}, \forall k \in \mathcal{K} \\
& {\left[\Re\left(\lambda_{k}\right)-t\right] \tan \theta_{t} \geq\left|\Im\left(\lambda_{k}\right)\right|, \forall k \in \mathcal{K}} \\
& \left\|\mathbf{F}_{r} \mathbf{y}_{r}\right\| \leq P_{r}
\end{array}
$$

which is convex and can be solved accordingly. We summarize the decoupled approach in Algorithm 2.

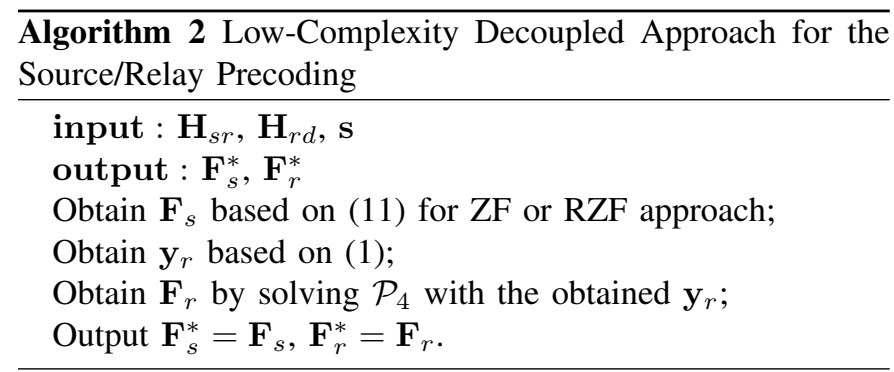

\section{NumERICAL RESUlTS}

In this section the numerical results based on Monte Carlo simulations are presented to evaluate the performance of the proposed precoding techniques. Throughout the simulations, we assume that QPSK modulation is employed, and the number of antennas at the relay is $N_{R}=4$. There is a total number of $K=4$ users in the cooperative system. For simplicity we assume $P_{s}=P_{r}=1, \sigma_{r}^{2}=\sigma_{d}^{2}=\sigma^{2}$, and the transmit SNR at the BS is accordingly defined as $\rho=1 / \sigma^{2}$. For comparison, we also depict the schemes where the relay employs the closed-form ZF or RZF precoding to show the gains for interference exploitation.

In Fig. 3, the convergence of the proposed joint scheme in Algorithm 1 is validated, where the accuracy threshold is $\delta_{t h}=10^{-3}$. As can be observed, the proposed alternating

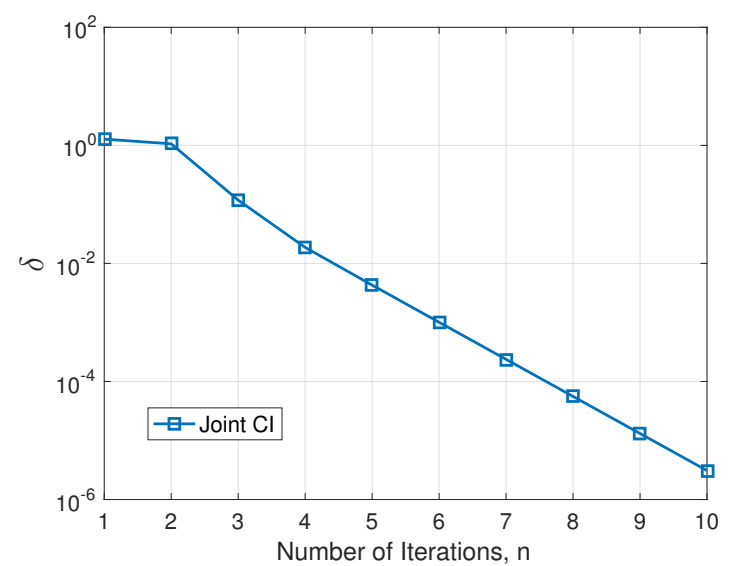

Fig. 3: Convergence validation, $\delta$ v.s. number of iterations $n$, $N_{t}=4, N_{R}=4, K=4, \delta_{t h}=10^{-3}$

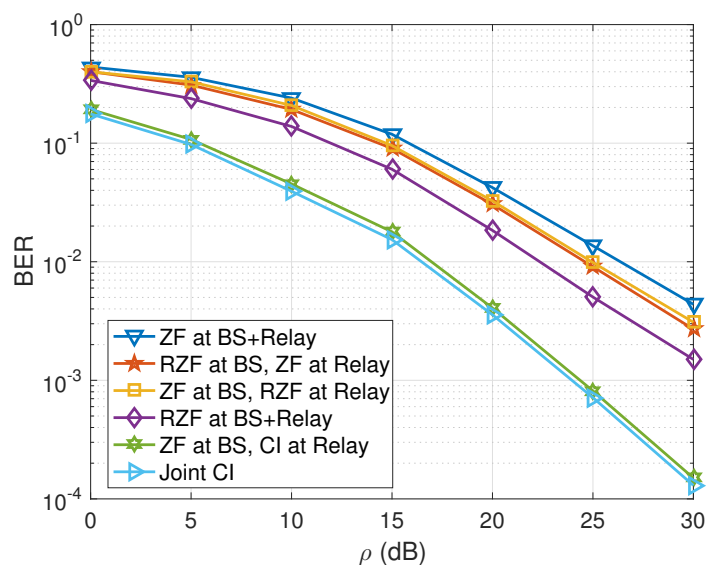

Fig. 4: BER v.s. transmit SNR, $N_{t}=4, N_{R}=4, K=4$, QPSK

optimization algorithm is shown to be convergent within in $n=7$ iterations.

In Fig. 4, we compare the bit error rate (BER) performance of the proposed interference exploitation techniques with conventional closed-form $\mathrm{ZF} / \mathrm{RZF}$ precoding techniques, where the closed-form relay precoding approaches follow (11) with the scaling factor satisfying the relay power constraint in (4). As expected, the proposed symbol-level precoding that exploits the constructive interference is more superior than the closed-form $\mathrm{ZF} / \mathrm{RZF}$ precoding approach, while generally it is observed that employing RZF instead of $\mathrm{ZF}$ precoding can further improve the BER performance.

In Fig. 5, we present the BER result of different schemes with a larger number of antennas $N_{t}=6$ at the BS. Compared to the extreme case shown in Fig. 4, it is observed that when $N_{t}$ is larger the performance is dominated by the precoding method employed at the relay, while the precoding scheme employed at the BS only has a marginal effect on the BER result. It is not surprising to observe that the proposed 


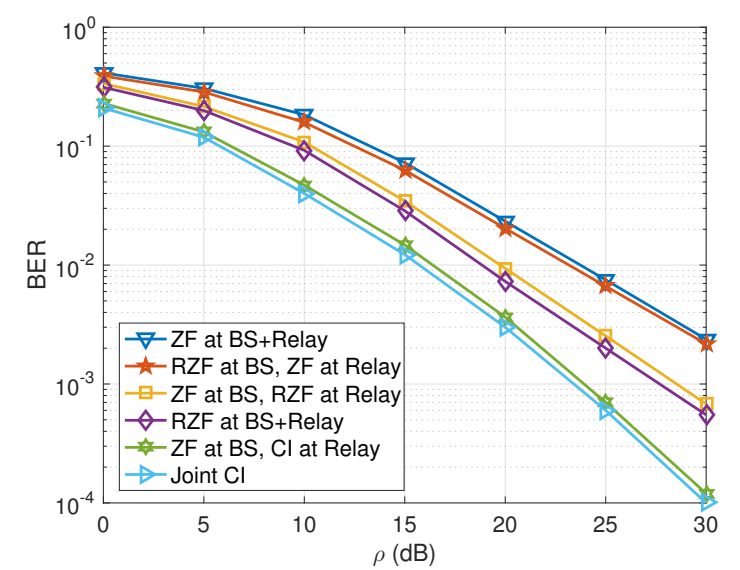

Fig. 5: BER v.s. transmit SNR, $N_{t}=6, N_{R}=4, K=4$, QPSK

techniques still achieve an improved performance.

\section{CONClusion}

In this paper, we study the downlink precoding techniques for a dual-hop AF relay assisted cooperative communication system. We propose both a joint source/relay precoding approach and a low-complexity decoupled precoding method by exploiting the symbol-level constructive interference. Both proposed techniques are shown to be advantageous over the conventional ZF or MMSE schemes. Moreover, compared to the joint approach that requires an alternating optimization process, the proposed decoupled scheme is shown to be low-complexity and able to achieve a similar performance, which favours its usefulness in practical wireless cooperative systems.

\section{ACKNOWLEDGMENT}

This work was supported by the Royal Academy of Engineering, UK, the Engineering and Physical Sciences Research Council (EPSRC) project EP/M014150/1, and the China Scholarship Council (CSC).

\section{REFERENCES}

[1] J. Mietzner, R. Schober, L. Lampe, W. H. Gerstacker, and P. A. Hoeher, "Multiple-Antenna Techniques for Wireless Communications - A Comprehensive Literature Survey," IEEE Commun. Surveys Tuts., vol. 11, no. 2, pp. 87-105, 2nd Quart. 2009.

[2] C.-B. Chae, A. Forenza, R. W. Heath, M. R. McKay, and I. B. Collings, "Adaptive MIMO Transmission Techniques for Broadband Wireless Communication Systems," IEEE Commun. Mag., vol. 48, no. 5, pp. 112-118, May 2010.

[3] J. Andrews, S. Buzzi, W. Choi, S. Hanly, A. Lozano, A. Soong, and J. Zhang, "What will 5G be?" IEEE J. Sel. Areas Commun., vol. 32, no. 6, pp. 1065-1082, June 2014.

[4] A. Nosratinia, T. E. Hunter, and A. Hedayat, "Cooperative Communications in Wireless Networks," IEEE Commun. Mag., vol. 42, no. 10, pp. 74-80, Oct. 2004.

[5] B. Wang, J. Zhang, and A. Host-Madsen, "On the Capacity of MIMO Relay Channels," IEEE Trans. Inf. Theory, vol. 51, no. 1, pp. 29-43, Jan. 2005.

[6] N. Yang, P. L. Yeoh, M. Elkashlan, J. Yuan, and I. B. Collings, "Cascaded TAS/MRC in MIMO Multiuser Relay Networks," IEEE Trans. Wireless Commun., vol. 11, no. 10, pp. 3829-3839, Oct. 2012.
[7] N. Yang, M. Elkashlan, P. L. Yeoh, and J. Yuan, "Multiuser MIMO Relay Networks in Nakagami-m Fading Channels," IEEE Trans. Commun., vol. 60, no. 11, pp. 3298-3310, Nov. 2012.

[8] H. Bolcskei, R. U. Nabar, O. Oyman, and A. J. Paulraj, "Capacity Scaling Laws in MIMO Relay Networks," IEEE Trans. Wireless Commun., vol. 5, no. 6, pp. 1433-1444, June 2006.

[9] Y. Fan and J. Thompson, "MIMO Configurations for Relay Channels: Theory and Practice," IEEE Trans. Wireless Commun., vol. 5, no. 5, pp. 1774-1786, May 2007.

[10] X. Tang and Y. Hua, "Optimal Design of Non-Regenerative MIMO Wireless Relays," IEEE Trans. Wireless Commun., vol. 6, no. 4, pp. 1398-1407, Apr. 2007.

[11] O. Munoz-Medina, J. Vidal, and A. Augstin, "Linear Transceiver Design in Nonregenerative Relays with Channel State Information," IEEE Trans. Sig. Process., vol. 55, no. 6, pp. 2593-2604, June 2007.

[12] T. Zeng, Q. Chen, P. Xiao, and J. Wu, "MSINR based Precoding Design for Non-Regenerative MIMO Relay System," in 2012 1st IEEE International Conference on Communications in China (ICCC), Beijing, 2012, pp. 508-513.

[13] R. Wang and M. Tao, "Joint Source and Relay Precoding Designs for MIMO Two-Way Relaying based on MSE Criterion," IEEE Trans. Sig. Process., vol. 60, no. 3, pp. 2593-2604, June 2012.

[14] C. T. Lin, F. S. Tseng, and W. R. Wu, "Limited-Feeback Precoding for Dual-Hop MIMO Relay Systems with SIC Receivers," IEEE Trans. Veh. Tech., vol. 65, no. 12, pp. 10142-10 147, Dec. 2016.

[15] W. Xu, X. Dong, and W. S. Lu, "MIMO Relaying Broadcast Channels with Linear Precoding and Quantized Channel State Information Feedback," IEEE Trans. Sig. Process., vol. 58, no. 10, pp. 5233-5245, Oct. 2010.

[16] S. Gong, C. Xing, N. Yang, Y. C. Wu, and Z. Fei, "Energy Efficient Transmission in Multi-User MIMO Relay Channels with Perfect and Imperfect Channel State Information," IEEE Trans. Wireless Commun., vol. 16 , no. 6, pp. 3885-3898, June 2017.

[17] C. Masouros and E. Alsusa, "Dynamic Linear Precoding for the Exploitation of Known Interference in MIMO Broadcast Systems," IEEE Trans. Wireless Commun., vol. 8, no. 3, pp. 1396-1404, March 2009.

[18] C. Masouros, "Correlation Rotation Linear Precoding for MIMO Broadcast Communications," IEEE Trans. Sig. Process., vol. 59, no. 1, pp. 252-262, Jan. 2011.

[19] C. Masouros and G. Zheng, "Exploiting Known Interference as Green Signal Power for Downlink Beamforming Optimization," IEEE Trans. Sig. Process., vol. 63, no. 14, pp. 3628-3640, July 2015.

[20] G. Zheng, I. Krikidis, C. Masouros, S. Timotheou, D. A. Toumpakaris, and Z. Ding, "Rethinking the Role of Interference in Wireless Networks," IEEE Commun. Mag., vol. 52, no. 11, pp. 152-158, Nov. 2014.

[21] C. Masouros, T. Ratnarajah, M. Sellathurai, C. B. Papadias, and A. K. Shukla, "Known Interference in the Cellular Downlink: A Performance Limiting Factor or a Source of Green Signal Power?" IEEE Commun. Mag., vol. 51, no. 10, pp. 162-171, Oct. 2013.

[22] M. Alodeh, S. Chatzinotas, and B. Ottersten, "Constructive Multiuser Interference in Symbol Level Precoding for the MISO Downlink Channel," IEEE Trans. Sig. Process., vol. 63, no. 9, pp. 2239-2252, May 2015.

[23] A. Li, C. Masouros, F. Liu, and A. L. Swindlehurst, "Massive MIMO 1Bit DAC Transmission: A Low-Complexity Symbol Scaling Approach," arXiv preprint, Available online: https://arxiv.org/abs/1709.08278.

[24] A. Li and C. Masouros, "Exploiting Constructive Mutual Coupling in P2P MIMO by Analog-Digital Phase Alignment," IEEE Trans. Wireless Commun., vol. 16, no. 3, pp. 1948-1962, March 2017.

[25] — , "Interference Exploitation Precoding Made Practical: ClosedForm Solutions with Optimal Performance," arXiv preprint, Available online: https://arxiv.org/abs/1712.07846.

[26] C. B. Peel, B. M. Hochwald, and A. L. Swindlehurst, "A VectorPerturbation Technique for Near-Capacity Multiantenna Multiuser Communication-Part I: Channel Inversion and Regularization," IEEE Trans. Commun., vol. 53, no. 1, pp. 195-202, Jan. 2005.

[27] L. Vandenberghe and S. Boyd, Convex Optimization. Cambridge University Press, 2004.

[28] M. Razaviyayn, M. Hong, and Z.-Q. Luo, "A Unified Convergence Analysis of Block Successive Minimization Methods for Nonsmooth Optimization,” SIAM J. Optim., vol. 23, no. 2, pp. 1126-1153, 2013. 